
matérielle de l'éducation

\title{
Le film fixe Pathéorama (1921) ou généalogie d'une invention
}

\section{Valérie Vignaux}

\section{(2) OpenEdition \\ Journals}

Electronic version

URL: http://journals.openedition.org/trema/3128

DOI: $10.4000 /$ trema.3128

ISSN: 2107-0997

\section{Publisher}

Faculté d'Éducation de l'université de Montpellier

\section{Printed version}

Date of publication: 1 June 2014

Number of pages: $36-43$

ISBN: 1167-315X

ISSN: $1167-315 X$

\section{Electronic reference}

Valérie Vignaux, «Le film fixe Pathéorama (1921) ou généalogie d’une invention », Tréma [Online],

41 | 2014, Online since 01 June 2016, connection on 20 April 2019. URL : http://

journals.openedition.org/trema/3128 ; DOI : 10.4000/trema.3128

This text was automatically generated on 20 April 2019.

Trema 


\title{
Le film fixe Pathéorama (1921) ou généalogie d'une invention
}

\author{
Valérie Vignaux
}

1 Le film fixe est une pellicule argentique de format $35 \mathrm{~mm}$, d'environ un mètre, sur lequel ont été reproduites et mises bout à bout une vingtaine voire une cinquantaine d'images. Son apparition dans l'histoire des projections d'images n'est pas datée, il semble toutefois émerger au début du XXe siècle, afin de remplacer les vues sur verre qui agrémentaient tout au long du XIXe siècle les conférences accompagnées par la projection. Il paraît tomber en désuétude aux alentours des années 1980, probablement supplanté par la diapositive qui permettait à l'usager de s'émanciper de la continuité imposée par la bande pelliculaire. Ainsi, pour concourir aux rares études qui voient le jour autour du film fixe ${ }^{1}$, j'ai choisi, parmi les milliers de films et les dizaines, voire les centaines, d'appareils qui ont circulé sur le territoire national au cours de cette période de m'intéresser au Pathéorama ${ }^{2}$. Il m'a semblé en effet, en l'absence de contre exemple, que le procédé mis sur le marché par la société Pathé à partir de 1921 était probablement à l'origine du dispositif. À partir des archives conservées, on s'efforcera de restituer le contexte dans lequel apparait l'appareil, et ce afin d'entreprendre la généalogie d'une invention.

\section{Le Pathéorama, un appareil de vision}

2 L'appareil n'est sans doute pas totalement finalisé lorsque la société Pathé dépose auprès de l'office national de la propriété industrielle un premier brevet le 25 mars 1921, car il sera complété par un additif et un autre brevet. Néanmoins, l'ensemble du dispositif est manifestement déjà conçu, l'appareil y est défini en fonction de trois paramètres : « [...] un film composé de vues cinématographiques diverses [...] ainsi qu'un appareil utilisable pour la vision directe du dit film et éventuellement pour sa projection ${ }^{3} »$. Le terme Pathéorama recouvre donc autant la bande pelliculaire « un film », qu'un appareillage de vision directe, c'est-à-dire individuelle, bientôt assorti d'une lanterne de projection, pour une vision collective. L'appareil à vision directe est : 
«[...] Une boîte rectangulaire [d'environ $14 \mathrm{~cm}$ x $6 \mathrm{~cm}$ ] à trois compartiments, dont les deux compartiments extrêmes [...] servent de magasin pour le film, et dont le compartiment médian forme une chambre noire, oblongue, comportant d'un côté la fenêtre ajourée qui permet d'éclairer le film par transparence, et de l'autre la loupe de vision ${ }^{4} »$.

Le brevet pour la lanterne ne sera déposé qu'en octobre 1922, soit un an après. Baptisée Cocorico, elle permet «à tous les possesseurs d'appareils à vision directe des bandes d'images de pouvoir transformer instantanément ces derniers en appareil de projection ${ }^{5}$ ». Elle n'autorise néanmoins qu'une projection de taille modeste, puisque d'après les catalogues, l'image ne peut dépasser un mètre de base sur quatre-vingt centimètres de hauteur. L'appareil qui s'apparente à une lanterne magique appartiendrait donc à la longue liste des jouets optiques qui fleurissent au début du XXe siècle, et ne constituerait qu'un appareil parmi d'autres. Il semble néanmoins, à la lecture du brevet, que sa singularité repose sur l'emploi d'une pellicule filmique car celle-ci est décrite avec une grande précision :

«Le film objet de l'invention est réalisé par un assemblage successif de vues cinématographiques diverses appartenant, ou non, à un même sujet et séparées éventuellement par des titres explicatifs [...]. Ce film peut se trouver composé de vues n'ayant entre elles aucune corrélation définie, de même qu'il peut se trouver composé de vues ayant entre elles une certaine corrélation; [...] ces vues peuvent illustrer les points principaux d'un sujet ou scène cinématographique et en donner une sorte d'abrégé [...] Bien que, pour la fabrication du dit film composé, on utilise principalement les nombreux déchets obtenus dans les fabriques de films cinématographiques [...] il est évident qu'on peut utiliser aussi les films usuels en séparant les vues dont ils sont composés et en les assemblant à nouveau entre elles $[\ldots]^{6} »$.

L'appareil participe donc d'une stratégie visant à substituer aux vues sur verre des images fixes sur pellicule, ce que confirmerait la déclaration faite aux actionnaires au cours d'une Assemblée générale réunie le 16 septembre 1920 où Charles Pathé précisait ses intentions :

"Nous vous informons que nous avons à l'étude actuellement un cinématographe populaire, d'un prix très minime et de dimensions réduites, que nous croyons susceptible d'être utilisé à la fois comme jouet pour les enfants, et comme appareil de démonstration pour certaines catégories d'enseignement. [...] Mais en tout état de cause, j'estime que si ce nouvel appareil, sur le lancement duquel nous comptons pour écouler le supplément de production de notre film vierge, venait à faire défaut, nous n'aurions aucune difficulté à utiliser notre pellicule dans les applications nombreuses que le film peut trouver dans le remplacement des plaques photographiques en verre employées pour la photographie ordinaire ${ }^{7}$ ».

Proposition qu'on retrouve clairement formulée dans le catalogue Pathéorama daté de 1926:

«Le but poursuivi par notre société a été de mettre à la portée de tous, pour un prix modique, un choix de vues sélectionnées avec soin, accompagnées d'un texte explicatif documenté, et pouvant rendre les mêmes services que les clichés sur verre, d'usage général mais de manipulation délicate en raison de leur fragilité ${ }^{8}$ ».

Le Pathéorama serait donc le premier appareil à vues fixes employant un support pelliculaire et l'examen des revues comme le Fascinateur et le Cinéopse susceptibles de présenter du matériel pédagogique sur la période de 1901 à 1920, paraît le confirmer puisqu'aucun autre dispositif comparable n'est présenté. Procédé qui sera très largement adopté par la plupart des fabricants occasionnant le remplacement progressif des vues 
sur verre, lourdes et fragiles, par ces petits rouleaux de pellicule, légers et peu encombrants. Charles Pathé qui possède une usine de pellicule entendait sans doute créer de nouveaux débouchés à sa production puisque les films fixes, bande d'un mètre environ, pouvaient être réalisés à partir des « déchets obtenus dans les fabriques de films ${ }^{9}$ ». L'additif au brevet déposé le 21 avril 1921, soit quelques semaines après, s'attache alors à définir et donc à concevoir la bande filmée elle-même. Dorénavant dénommée « film composé », celle-ci comprend des vues qui peuvent être associées.

Elle est «constituée par une pellicule cinématographique vierge sur laquelle on aura reproduit les sujets désirés, que ces sujets soient choisis parmi les images de films cinématographiques ou bien parmi des collections de vues photographiques ${ }^{10} »$.

Cette définition suggère deux types d'emplois. Si les images sont empruntées aux « films cinématographiques", possiblement de fiction, elles constituent alors un spectacle récréatif; tandis qu'en puisant dans les "collections de vues photographiques", composés de la sorte à partir de clichés documentaires, les bandes pourraient avoir valeur instructive. Le terme de " collection » paraît se référer aux recueils thématiques destinés à illustrer les conférences d'éducation populaire et constitués par les associations, privées ou publiques, qui officiaient depuis la fin du XIXe siècle à travers le territoire national. Le Pathéorama apparait comme un objet ambivalent: son maniement simplifié et léger, individuel, l'apparente à un jouet pour enfant; alors que les films qu'ils soient de fiction ou documentaires, paraissent viser un public adulte, pour des séances collectives. Le Pathéorama a-t-il été conçu pour le marché familial ou éducatif?

\section{Le Pathéorama, un appareil pour quels usages ?}

9 Le Pathéorama est un objet au coût modeste, l'appareil de vision directe est vendu une dizaine de francs et les films sont cédés entre 2 francs cinquante et 5 francs, ce qui le met à la portée d'un public populaire. Par contre l'acquisition du projecteur Cocorico pour la somme de 120 francs constitue certainement une dépense plus importante et il existe aussi une version en bakélite, dans une matière plus luxueuse, de l'appareil de vision directe. Lorsqu'il présente l'appareil pour en faire la promotion, l'industriel le met aux mains de femmes ou d'enfants, sans doute afin d'affirmer la simplicité de son maniement. Positionnement familial qui place alors le Pathéorama dans la lignée des inventions imaginées par Charles Pathé afin de développer le marché privé. Dès 1912, l'industriel a mis sur le marché une caméra assortie d'un appareil de projection employant une pellicule de $28 \mathrm{~mm}$, soit d'un format moindre et donc d'un coût moins élevé, baptisée Pathé-Kok. Procédé qui est abandonné sans doute en raison de la Première Guerre mais aussi parce que son prix trop élevé empêche l'expansion du marché privé. Au début des années 1920, lorsque paraît le Pathéorama, Charles Pathé s'est à nouveau concentré sur le marché amateur et le 21 février 1921, il a déposé les plans d'une caméra et d'un appareil de projection dénommés Pathé-Baby, conçus pour employer une pellicule au format 9,5 $\mathrm{mm}$ à perforation centrale. Dans le même temps, le 16 septembre 1920, Charles Pathé a fait ratifier au cours d'une assemblée générale un éclatement de sa société en deux entités. La première, Pathé-Consortium, s'est vue transférer le stock des films et l'ensemble des activités de production, de distribution et d'exploitation. La seconde, Pathé-Cinéma, se voit confier la fabrication de la pellicule vierge, la construction du matériel, le cinéma de format réduit pour amateurs et l'exploitation familiale ou le cinéma scolaire. Charles Pathé déclare se désintéresser de la première et place l'essentiel 
de ses intérêts économiques dans la seconde. Le Pathéorama s'inscrit donc dans les initiatives qui s'emploient à accroître les pratiques privées mais l'industriel a néanmoins d'autres visées sinon l'appareil viendrait doubler le Pathé-Baby, sans parvenir à l'égaler. Charles Pathé, pensait sans doute, en supplantant la fabrication de vues sur verre, intéresser les spécialistes de la pédagogie par l'image car comme nombre de ses contemporains, il s'intéresse au cinéma d'enseignement. En 1923, il commercialise un projecteur dénommé Pathé-Enseignement et, en 1926, il lance une revue intitulée Le Cinéma chez soi, dont le comité de patronage comprend les noms des principaux promoteurs du cinéma éducateur. On y retrouve Jules Breton, ministre de l'Hygiène et président de la fameuse commission extra-parlementaire dite Bessou, chargée en 1916 par Paul Painlevé, alors ministre de l'Instruction publique, d'étudier les moyens de généraliser l'emploi du cinéma dans l'enseignement. À ses côtés se trouvent également Julien Luchaire, directeur de l'Institut de coopération intellectuelle, Henri Queuille, protecteur de la cinémathèque du ministère de l'Agriculture, Edmond Labbé, protecteur de la cinémathèque de l'Enseignement professionnel et le sénateur Joseph Brenier, président de l'office du cinéma éducateur de Lyon ${ }^{11}$. L'éditorial inaugural justifie le projet en ces termes: «Notre titre contient tout notre programme. Nous nous adressons à la famille et à cette autre famille qu'est l'école ». Or, dès juin-juillet 1927, soit à peine un an après son lancement, le changement de maquette augure une modification certaine quant aux premières visées :

«La formule de ce journal sera désormais élargie. Nous laisserons certes au cinéma éducatif sa large part [...] Notre revue demeurera celle de la famille et de l'école. Toutefois, la vie cinématographique diverse, active, universelle sera plus abondamment commentée. Nous ferons connaître les projets des auteurs, les réalisations des studios, les créations des artistes ${ }^{12} »$.

10 La revue abandonne ses ambitions didactiques, et se transforme en organe d'information à destination des usagers du Pathé-Baby. Elle paraît destinée aux amateurs mais aussi aux filiales qui distribuent à travers le territoire les appareils et les films dont les adresses sont régulièrement rappelées. On trouve encore jusqu'en 1932 au sein de la revue des publicités en faveur du Pathéorama.

11 La conception du Pathéorama aurait donc trois objectifs : créer de nouveaux débouchés pour la pellicule vierge, familiariser un public de non-professionnels à la projection d'images et mettre à la disposition des pédagogues de tous ordres, mères de famille, instituteurs ou prêtres, des vues susceptibles de leur convenir. La présentation du catalogue daté de 1926 reprend l'argument: "Notre collection de films qui atteint le chiffre de 300 constitue une véritable encyclopédie qui doit être conservée et qui [...] mérite d'être consultée souvent ${ }^{13} »$. En se réclamant de l'Encyclopédie, Charles Pathé s'inscrit dans un courant d'idées qui depuis les Lumières valorise l'emploi des images dans la transmission des connaissances ${ }^{14}$. Une publicité, parue dans Le Cinéma chez soi en 1929 précise la composition du catalogue :

«Le choix des films est très grand et comporte des vues documentaires [...], des vues des colonies, des voyages et aussi des principales scènes tirées des grands films. Les films religieux ne sont pas oubliés [...] de même que les contes enfantins ${ }^{15}$ ».

12 Les films consultés sont agencés à partir d'une quarantaine d'images fixes. Ils sont parfois composés de plusieurs parties intitulées "séries", en référence très probablement au terme de « sérial » employé dans l'industrie cinématographique d'alors. Les films qui font la notoriété de la société comme Les Trois mousquetaires, film en douze épisodes réalisé 
par Henri Diamant-Berger (1921), L'Arlésienne (1921) d'André Antoine, L'Empereur des pauvres de René Leprince (1922) ou L'Enfant roi de Jean Kemm (1923) ont été adaptés en deux ou trois épisodes et probablement réalisés à partir des fameux "déchets cinématographiques ». Le film de Robert Flaherty Nanouk, d'une durée de 55 minutes, a pour sa part été condensé en trois épisodes d'une quarantaine d'images chacun. Des éléments descriptifs ont été écartés, les intertitres transformés, toutefois les œuvres sont manifestement respectées dans leur structure et leur continuité. Les adaptations cinématographiques représentent 29 titres sur un ensemble de 277 , soit près de $11 \%$ du catalogue, en nombre donc moins élevé que les vues documentaires, qui avec 141 titres représentent $51 \%$ de l'ensemble. Ces vues documentaires sont constituées de films de " tourismes », « grandes villes ", « montagne ", « plages et ports », « colonies françaises » mais aussi «actualités». Les sujets sont agencés à partir de productions existantes, comme le film réalisé à partir d'une "Cinégraphie» de R. Alexandre, mais ils peuvent aussi être composés au moyen de vues photographiques commercialisées par d'autres firmes ou organismes, tels que les clichés Le Deley, les Clichés d'Art de l'Agence économique du Gouvernement Général de l'Afrique Occidentale française - qui distribue aussi des films -, les images empruntées au Verascope Richard plus connu pour son procédé de photographie en relief, ou les cartes postales des éditions L'Abeille, de la Librairie Blanchard ou du C.A.P. Strasbourg. Sous l'intitulé documentaire se côtoient donc des images extrêmement hétérogènes. La rubrique associe aux films de tourisme des sujets consacrés aux événements comme Les Fêtes de la Victoire 1919 ou L'Exposition internationale des arts décoratifs de 1925 qui auraient pu être classés dans la rubrique Actualités. Les vues peuvent faire l'objet de plusieurs montages et les films intitulés En Tunisie et La Vie au Harem reprennent les mêmes visages de femmes.

Les films pour enfants, qui représentent 61 titres sur 277 , soit $22 \%$, sont là encore conçus à partir d'images issues de registres divers: imagerie d'Epinal pour une adaptation du Petit Poucet, montage d'un dessin animé de Roger Lortac Un chien trop bien dressé, ou encore composition à partir de photographies pour un "film comique», le seul de sa rubrique, intitulé Toby chien garde l'auto. Les films religieux, au nombre de $12(4,5 \%)$, peuvent être des adaptations, comme cette Vie de Jésus en quatre parties d'une quarantaine d'images chacune : Naissance, Enfance, Miracles et Vie publique, Passion et mort, dont je n'ai pu retrouver avec certitude l'auteur mais qui est très probablement adaptée de la Passion réalisée par Ferdinand Zecca en 1904. Ou encore ce film intitulé Lourdes ou les apparitions de la Sainte Vierge à Bernadette, réalisé à moindre frais puisque composé à partir de deux cadres seulement. Catalogue qui malgré les déclarations, n'a rien d'encyclopédique, il n'existe par exemple aucun film à caractère scientifique, et les vues sont en majorité pittoresques ce qui souligne leur ambition distractive.

\section{Ambivalence d'une invention}

14 S'il fut un temps pionnier sur le marché, le Pathéorama est rapidement concurrencé et on dénombre dès la fin des années 1930 une dizaine de modèles de projecteurs "fixes ». Encore commercialisé jusqu'au milieu des années 1930, l'appareil paraît tomber en désuétude, sans doute en raison de son catalogue, à la fin de la décennie. La technique a néanmoins fait ses preuves, et elle prendra son essor aux lendemains de la Seconde Guerre, sous l'effet de la loi Barangé16, loi qui accordait aux établissements scolaires 
d'importants crédits pour l'achat de matériel de projection. L'appareil Pathéorama, manifestement le premier du genre, est un objet charnière, situé entre deux siècles et entre deux techniques, entre la lanterne magique et le cinéma. Pour autant, en raison des visées « encyclopédiques» de son catalogue, il rappelle aussi le fait que depuis le XVIIIe siècle, la projection des images s'inscrit dans des processus de transmission des connaissances. Sa disparition pourrait s'expliquer par son ambivalence, le Pathéorama est en effet conçu à la fois pour une vision individuelle et collective, pour des usages ludiques ou instructifs, pour la famille ou l'école, équivoque dont témoigne aussi son catalogue. L'appareil viendrait confirmer la proposition d'André Gaudreault et Philippe Marion lorsqu'ils déclarent qu'un média nait toujours deux fois :

"Lorsqu'un média apparait, il existe déjà un intelligible médiatique. Lorsqu'un média arrive au monde il doit aussi se colleter avec un code déjà établi (des genres, des institutions d'autres médias, etc.). Notre propos voudrait montrer combien est problématique et paradoxale l'idée même de naissance d'un média, du moins si l'on entend par naissance l'apparition d'un événement unique, circonscrit, ponctuant avec précision la chaine historique. Car un média ne s'impose réellement en tant que média autonome, avéré et digne de ce nom que lorsqu'il a rendu tangible et crédible sa part d'opacité propre. C'est-à-dire sa manière propre de représenter, d'exprimer et de communiquer le monde ${ }^{17}{ }^{\prime}$.

L'itinéraire historique de cette invention montre comment le procédé, s'il est à l'origine des appareils employant $d u$ film fixe n'en possède pas encore les caractéristiques utilitaires qui conduiront par la suite à son implantation sociale et industrielle. Ainsi, lorsque disparait le Pathéorama, le film fixe est encore à inventer.

\section{BIBLIOGRAPHY}

Gaudreault A., Marion P. (2000). Un média nait toujours deux fois. Société \& Représentations.

Lefebvre, T. (1997). Sage comme une image. L'abbé Songaylo et le Pathéorama. 1895, n²1.

Lefebvre, T. (2001). Film safety, formats réduits, films fixes. In Jeunet, P., Nourrisson, D., (dir.), Cinéma-école : aller-retour. Actes du colloque de Saint-Étienne. Novembre 2000. Saint-Étienne : Publications de l'Université de Saint-Étienne.

Lefebvre, T. (2001). Films fixes et santé publique. Revue d'histoire de la pharmacie, $n^{\circ} 331$.

Pathé, C. (2006). Écrits autobiographiques. Paris : L’Harmattan.

Nourrisson, D. (2001). Le 7e art... d'enseigner : le film fixe. In Jeunet, P., Nourrisson, D. (dir.), Cinéma-école : aller-retour. Actes du colloque de Saint-Étienne. Novembre 2000. Saint-Étienne : Publications de l'Université de Saint-Étienne.

Nourrisson, D. (2002). Arrêt sur les images de l'alcoolisme. In Nourrisson, D. (dir.), À votre santé ! Éducation et santé sous la 4e République. Saint-Etienne : Presses Universitaires de SaintEtienne. 
Vignaux, V. (2010). Le film fixe Pathéorama (1921) phénomène ou paradigme ? In Lugon, O., Guido, L. (dir.), Fixe/Animé : croisements de la photographie et du cinéma au XXe siècle. Lausanne : L'Âge d'Homme.

Vignaux, V. (2007). Jean Benoit-Lévy ou le corps comme utopie, une histoire du cinéma éducateur dans l'entre-deux-guerres en France. Paris : AFRHC.

\section{NOTES}

1. Cf. bibliographie en fin d'étude.

2. Cet article reprend une précédente étude intitulée: Vignaux, V. (2010). Le film fixe Pathéorama (1921) phénomène ou paradigme? In Lugon, O., Guido, L. (dir.), Fixe/Animé : croisements de la photographie et du cinéma au XXe siècle. Lausanne: L'Âge d'Homme, pp. 133-144. Et traduction anglaise : Betwin Still and Moving Images, John Libbey Publishing Ltd, 2012.

3. «Brevet $n^{\circ} 532.804$, Office national de la propriété industrielle, République française», conservé à la Cinémathèque française, collection des appareils.

4. Idem.

5. «Brevet $n^{\circ} 534.375$, Office national de la propriété industrielle, République française», conservé à la Cinémathèque française, collection des appareils.

6. Idem.

7. Cf. Pathé, C. (2006). Écrits autobiographiques. Paris : L'Harmattan. Édition établie, annotée et présentée par Pierre Lherminier, collection Les temps de l'image, p. 299.

8. Catalogue Pathéorama, 1926, p. 2, Fondation Jerôme Seydoux-Pathé.

9. Idem.

10. «Brevet $n^{\circ} 24.748$, 1re addition au brevet d'invention $n^{\circ} 532.804$, office national de la propriété industrielle, République française ", Cinémathèque française, collection des appareils.

11. Personnalités et commissions dont les activités sont détaillées dans Vignaux, V. (2007). Jean Benoit-Lévy ou le corps comme utopie, une histoire du cinéma éducateur dans l'entre-deuxguerres en France. Paris : AFRHC.

12. Cinéma chez soi, juin-juillet 1927.

13. Catalogue Pathéorama, 1926, p. 2, archive citée.

14. Cf. Barthes R. (1964). Image, raison, déraison. In Barthes, R., Mauzi, R. et Seguin, J.-P. (dir.), L'Univers de l'Encyclopédie. Paris : Les Libraires associés, reproduit dans Serri, J. (1989). Les Planches de l'Encyclopédie de Diderot et d'Alembert vues par Roland Barthes, catalogue exposition au Musée de Pontoise, édition Association les amis de Jeanne et Otto Feundlich.

15. Le Cinéma chez soi, $n^{\circ} 28$, février 1929 , non paginé.

16. Cf. Lefebvre, T. (2001). Film safety, formats réduits, films fixes. In Jeunet, P., Nourrisson, D., (dir.), Cinéma-école : aller-retour. Actes du colloque de Saint-Étienne. Novembre 2000. SaintÉtienne : Publications de l'Université de Saint-Étienne.

17. Gaudreault, A., Marion, P. (2000). Un média nait toujours deux fois. Société \& Représentations, p. 22. 


\section{ABSTRACTS}

This study would like to reconstruct the history of the Pathéorama filmstrip, from the preserved archives. This paper would like both to clarify the history of an invention and suggest ideas for a cultural history of film techniques.

Cette étude s'attache à restituer, à partir des archives conservées, l'histoire du film fixe Pathéorama. Cette contribution souhaite à la fois préciser l'histoire d'une invention et proposer des perspectives pour une histoire culturelle des techniques cinématographiques.

\section{INDEX}

Mots-clés: Charles Pathé, cinéma éducateur, film fixe, Pathéorama

Keywords: educator cinema, filmstrip

\section{AUTHOR}

\section{VALÉRIE VIGNAUX}

Maître de conférences en études cinématographiques habilitée à diriger les recherches, Équipe InTru (Interactions, transferts et ruptures artistiques et culturels), EA 6301, Université FrançoisRabelais de Tours 